\title{
ZEB1 overexpression associated with E-cadherin and microRNA-200 downregulation is characteristic of undifferentiated endometrial carcinoma
}

Laura Romero-Pérez ${ }^{1}$, M Ángeles López-García ${ }^{1}$, Juan Díaz-Martín ${ }^{1}$, Michele Biscuola ${ }^{1}$, M Ángeles Castilla ${ }^{1}$, Laura J Tafe ${ }^{2}$, Karuna Garg ${ }^{3}$, Esther Oliva ${ }^{4}$, Xavier Matias-Guiu ${ }^{5}$, Robert A Soslow ${ }^{3}$ and José Palacios ${ }^{6}$

${ }^{1}$ Department of Pathology, Instituto de Biomedicina de Sevilla (IBiS), Hospital Universitario Virgen del Rocío/ CSIC/Universidad de Sevilla, Sevilla, Spain; ${ }^{2}$ Department of Pathology, Darmouth-Hitchcock Medical Center, Lebanon, NH, USA; ${ }^{3}$ Department of Pathology, Memorial Sloan-Kettering Center, Memorial Hospital, New York, NY, USA; ${ }^{4}$ Department of Pathology, Massachusetts General Hospital, Boston, MA, USA; ${ }^{5}$ Department of Pathology and Molecular Genetics, Hospital Universitari Arnau de Vilanova, University of Lleida, IRBLleida, Lleida, Spain and ${ }^{6}$ Department of Pathology, Hospital Universitario Ramón y Cajal, Instituto de Investigación Sanitaria Ramón y Cajal (IRYCIS), Madrid, Spain

\begin{abstract}
Undifferentiated endometrial carcinomas are very aggressive high-grade endometrial carcinomas that are frequently under-recognized. This study aimed to analyze the molecular alterations underlying the development of these endometrial carcinomas, focusing on those related to dedifferentiation. We assessed a series of 120 tumors: 57 grade 1 and 2 endometrioid endometrial carcinomas, 15 grade 3 endometrioid endometrial carcinomas, 27 endometrial serous carcinomas, and 21 undifferentiated endometrial carcinomas. We found a high frequency of DNA mismatch repair deficiency $(38 \%)$ and moderate rate of p53 overexpression $(\sim 33 \%)$ in undifferentiated carcinomas. In contrast to the characteristic endometrioid phenotype, there was a dramatic downregulation of E-cadherin expression in the undifferentiated subtype. Quantitative methylation studies dismissed CDH1 promoter hypermethylation as the mechanism responsible for this change in gene expression, while immunohistochemistry revealed that the E-cadherin repressor ZEB1 was frequently overexpressed $(62 \%)$ in undifferentiated endometrial carcinomas. This finding was accompanied by a sharp downregulation in the expression of the miR-200 family of microRNAs, well-known targets of ZEB1. Furthermore, there was enhanced expression of epithelialto-mesenchymal transition markers in undifferentiated endometrial carcinomas, such as $\mathrm{N}$-cadherin, cytoplasmic p120, and osteonectin. In addition, HMGA2, a regulator of epithelial-to-mesenchymal transition that is expressed in aggressive endometrial tumors, such as endometrial serous carcinomas and carcinosarcomas, was expressed in $>20 \%$ of undifferentiated carcinomas. These results suggest that ZEB1 overexpression, associated with E-cadherin and miR-200s downregulation, and the expression of mesenchymal markers might enhance the metastatic potential of undifferentiated endometrial carcinomas, leading to a poor prognosis. In addition, our observations suggest that the immnohistochemical analysis of E-cadherin and ZEB1 can aid in the differential diagnosis of the more agressive undifferentiated endometrial carcinomas from grade 3 endometrioid carcinomas. Modern Pathology (2013) 26, 1514-1524; doi:10.1038/modpathol.2013.93; published online 7 June 2013
\end{abstract}

Keywords: E-cadherin; miR-200s; undifferentiated endometrial carcinoma; ZEB1

Correspondence: Dr RA Soslow, MD, Department of Pathology, Memorial Sloan-Kettering Center, Memorial Hospital, 1275 York Avenue, New York, NY 10065, USA.

E-mail: soslowr@mskcc.org

Dr J Palacios, MD, PhD, Department of Pathology, Hospital Universitario Ramón y Cajal, Instituto de Investigación Sanitaria Ramón y Cajal (IRYCIS), Carretera de Colmenar Viejo, km. 9,100, Madrid 28034, Spain.

E-mail: jose.palacios@salud.madrid.org

Received 25 January 2013; revised 10 April 2013; accepted 11 April 2013; published online 7 June 2013
Undifferentiated endometrial carcinoma is a poorly differentiated subtype of endometrial carcinoma. ${ }^{1}$ Altrabulsi et al. ${ }^{2}$ defined undifferentiated endometrial carcinoma as a 'tumor composed of medium or large size cells with a complete absence of glandular differentiation, and with absent or minimal $(<10 \%)$ neuroendocrine differentiation.' Undifferentiated endometrial carcinoma represents about $9 \%$ of all endometrial carcinomas. In some cases, these tumors 
develop as undifferentiated areas associated with grade 1 or 2 endometrioid endometrial carcinomas, and such cases are often referred to as dedifferentiated carcinomas. ${ }^{3}$ Nevertheless, despite these studies, this high-grade endometrial carcinoma remains underrecognized or/and it may be frequently classed as FIGO grade 3 endometrioid adenocarcinoma, ${ }^{4}$ even though undifferentiated endometrial carcinomas have a more aggressive behavior.

The molecular changes underlying undifferentiated endometrial carcinoma development are poorly understood. Although a high level of microsatellite instability has been reported, ${ }^{1,5}$ no studies have explored other molecular alterations that are common in endometrial carcinogenesis, including PTEN, CTNNB1, or TP53. Impaired differentiation is a key feature of undifferentiated endometrial carcinoma. Indeed, gland formation requires the presence of differentiated polarized epithelial cells in relation to adjacent cells. There are several mechanisms by which tumor cells could lose their ability to form glands, as seen in undifferentiated endometrial carcinoma. A frequent alteration observed in poorly differentiated carcinomas in any tissue involves a reduction in E-cadherin expression, a master regulator of cell adhesion and polarity encoded by the $C D H 1$ gene. ${ }^{6-8}$ Dampening E-cadherin expression can occur in several ways, transcriptional repression being one of the most important. Known repressors of E-cadherin include zinc-finger transcription factors (Snail1 (SNAI1), Slug/Snail2 (SNAI2), ZEB2 (SIP1), and ZEB1 $(\delta$-EF1)), and basic helix-loop-helix transcription factors (E47 (TCF3), E2-2 (TCF4), or Twist). E-cadherin downregulation through transcriptional repression is frequently associated with the epithelial-to-mesenchymal transition, a process of cellular transdifferentiation by which epithelial cells lose their polarity and cell-cell contacts, they reorganize their cytoskeleton, express mesenchymal markers, and they display a migratory phenotype. During epithelial-to-mesenchymal transition, progressive loss of E-cadherin expression is frequently coupled with the expression of non-epithelial cadherins, such as the mesenchymal $\mathrm{N}$-cadherin and cadherin-11, a process known as 'cadherin switching.' 9 In addition, cells acquire mesenchymal markers such as vimentin, fibronectin, SPARC, and others.

Recent reports have shown the importance of certain microRNAs (miRNAs) in modulating epithelial differentiation, and the miR-200 family appears to be a key regulator of this process. These miRNAs maintain the epithelial phenotype by downregulating the expression of E-cadherin repressors ZEB1 and ZEB2, which are directly targeted at their $3^{\prime} \mathrm{UTR}$ region. ${ }^{10-13}$ In turn, ZEB1 and ZEB2 bind to a conserved pair of ZEB-type E-box elements located at the promoter region of the miR-200s, both in the pri-miR-200a/b/429 ${ }^{13}$ and pri-miR-200c/141 loci. $^{12}$ Thus, a double-negative feedback loop exists between ZEB1/ZEB2 and miR-200s in epithelialto-mesenchymal transition regulation. ${ }^{13}$ Moreover, this loop has also been demonstrated to be crucial in stemness maintenance. ${ }^{14}$

To better understand the molecular mechanisms involved in undifferentiated endometrial carcinoma development and those underlying the lack of differentiation in this type of carcinoma, we analyzed the possible alterations in genes usually associated with endometrial carcinogenesis and those with the maintenance of the epithelial phenotype. For the first time, we show that undifferentiated endometrial carcinomas exhibit a complete loss or severe reduction in E-cadherin expression, which is associated with overexpression of E-cadherin repressor ZEB1 and downregulation of miR-200s. Furthermore, we show a molecular profile intermediate to that observed in endometrioid and serous carcinomas.

\section{Materials and methods}

\section{Patients and Tissues}

The present series consisted of 120 endometrial carcinomas: 57 grade 1 and 2 endometrioid endometrial carcinomas, 15 grade 3 endometrioid endometrial carcinomas, 27 endometrial serous carcinomas, and 21 undifferentiated endometrial carcinomas. Histological typing was performed by three pathologists (RAS, JP, and MAL-G) according to the World Health Organization (WHO) classification and previous reports on undifferentiated endometrial carcinomas. ${ }^{1,2,4}$ Tissue samples were obtained from the Department of Pathology at the Hospital Universitario Virgen del Rocio (Seville, Spain), the Department of Pathology at Memorial SloanKettering Cancer Center (New York, NY, USA), the Department of Pathology at Massachusetts General Hospital (Boston, MA, USA), and the Department of Pathology at the Hospital Universitari Arnau de Vilanova (IRBLleida, Lleida, Spain). A summary of the relevant clinicopathological findings is provided in Supplementary Table 1. The current study was approved by the relevant institutional committees.

\section{Tissue Microarray Construction and Immunohistochemistry}

Tissue sections $(5 \mu \mathrm{m})$ from 120 formalin-fixed paraffin-embedded samples were stained with hematoxylin and eosin, and representative areas were selected to obtain two 1-mm-diameter tissue cores. Tissue microarray sections were assessed by immunohistochemistry using the Envision method (Dako, CA, USA) and primary antibodies raised against MLH1, PMS2, MSH2, MSH6, $\beta$-catenin, p53, E-cadherin, P-cadherin, p120, N-cadherin, SPARC/ osteonectin, fascin, caveolin-1, HMGA2, and ZEB1. 
Immunohistochemical staining was evaluated by JP and MAL-G (antibody suppliers, dilutions, and the scoring criteria are shown in Supplementary Table 2).

\section{DNA Isolation and Sequenom MassARRAY Quantitative DNA Methylation Analysis}

DNA from 19 formalin-fixed paraffin-embedded tissues (10 undifferentiated endometrial carcinomas and 9 endometrioid endometrial carcinomas) was isolated with the QiAamp DNA Mini Kit (Qiagen, Hilden, Germany), determining the quantity and quality of DNA with a Nanodrop ND-2000 Spectrophotometer (Thermo Scientific, Waltham, MA, USA). Bisulfite conversion of DNA was performed using the EpiTect Plus DNA Bisulfite Kit (Qiagen) in a GeneAmp PCR 9700 system. Subsequently, the purified treated DNA was amplified in a TProfessional Standard 384 Thermocycler (Biometra, Jena, Germany) using specific PCR primers covering 343 bp in three amplicons involving $22 \mathrm{CpG}$ islands at the $C D H 1$ gene promoter region (for details of PCR primers and conditions see Supplementary Table 3). MassARRAY spectrometry of the PCR products was performed according to the manufactureŕs protocol and the data processed with EpiTyper Analyzer software v1.0 (Sequenom, San Diego, CA, USA).

\section{mRNA and miRNA Expression Analysis}

We analyzed gene and miRNA expression in 54 formalin-fixed paraffin-embedded samples of endometrial cancer (15 grade 1/2 endometrioid endometrial carcinomas, 4 grade 3 endometrioid endometrial carcinomas, 19 endometrial serous carcinomas, and 16 undifferentiated endometrial carcinomas), selecting samples where the tumor component represented over $70 \%$ of the cells. RNA from samples was isolated using the Recover All Total Nucleic Acid Isolation Kit (Ambion (Life Technologies), CA, USA) determining the quantity and quality of the total RNA on a Nanodrop ND-2000 Spectrophotometer (Thermo Scientific). We further assessed RNA quality using the RNA integrity value obtained from the Agilent 2100 Bioanalyzer (Agilent Technologies, CA, USA). After reverse transcription with the high capacity RNA to cDNA Kit (Applied Biosystems (Life Technologies), CA, USA), the cDNAs were preamplified following the TaqMan PreAmp Master Mix (Applied Biosystems, USA) protocol for the specific gene expression assays using a GeneAmp PCR 9700 system (Applied Biosystems) for both reverse transcription and cDNA preamplification. We designed Taqman Custom Array Plates (Applied Biosystems) for qRT-PCR of the mRNAs that contained primers for CDH1 (E-cadherin), E47/TCF3, HMGA2, SNAI1, SNAI2, E2-2/ TCF4, TGFb1, TGFb2, ZEB1, ZEB2, and the $18 S$ and GUSB endogenous controls (Supplementary Table 4).
qRT-PCR amplification was achieved using the SensiFAST Probe Hi-ROX mix (Bioline Reagents, London, UK) according to the manufactureŕs conditions.

We also used qRT-PCR to measure expression of the miR-200 family of miRNAs (miR-200a/b/c, miR-141, and miR-429) with RNU48 as an endogenous control (Supplementary Table 5). Prior reverse transcription was performed using the TaqMan MicroRNA Reverse Transcription Kit (Applied Biosystems) in the GeneAmp PCR 9700 system and qRT-PCR amplification with the TaqMan Universal PCR Master Mix (Applied Biosystems). All qRT-PCR measurements were obtained in a 7900HT Fast Real Time PCR System with the SDS 2.3 software (Applied Biosystems).

\section{Statistical Analysis}

Gene and miRNA expression data were normalized with the RQ Manager software, and analyzed with the SDS 2.3 software (Applied Biosystems) and Integromics RealTime StatMiner 4.1 package. This software performs a moderate $t$-test between two groups corrected with a Benjamini-Hochberg algorithm $(P<0.05)$. Normality tests to assess sample distribution, cross tables with Pearson's $\chi^{2}$ test to evaluate immunohistochemical differences, Pearson's correlations to determine association between mRNA and miRNA expression, and Student's $t$-test or one-way ANOVA to compare means of expression between groups were performed using SPSS 17.0 for Windows (SPSS). Significance was considered at $P \leq 0.05$.

\section{Results}

\section{Undifferentiated Endometrial Carcinomas are Characterized by a High Frequency of DNA Mismatch Repair Deficiency}

To determine the possible molecular events associated with undifferentiated endometrial carcinoma development, we studied the expression of some genes implicated in the initiation of endometrial carcinoma in both endometrioid (DNA repair proteins, CTNNB1, and PTEN) and non-endometrioid (TP53) tumors. The pattern of molecular changes in undifferentiated endometrial carcinoma resembled that of grade 3 endometrioid endometrial carcinoma, as in both tumor types the losses of DNA mismatch repair proteins was more common and p53 overexpression occurred moderately when compared with that in grade $1 / 2$ endometrioid endometrial carcinoma and endometrial serous carcinoma. By contrast, the frequency of $\beta$-catenin accumulation, an indicator of WNT pathway activation, was very low in undifferentiated endometrial carcinomas compared with all grades of endometrioid endometrial carcinomas (Table 1). We analyzed whether MSI (the 
Table 1 Immunohistochemical analysis of different markers in low-grade endometrioid endometrial carcinoma (EEC), high-grade endometrioid endometrial carcinoma (G3), endometrial serous carcinoma (ESC), and undifferentiated endometrial carcinoma (UEC) samples

\begin{tabular}{|c|c|c|c|c|c|c|}
\hline \multirow[b]{2}{*}{ Marker } & \multirow[b]{2}{*}{ Evaluation } & \multicolumn{4}{|c|}{ Phenotypes } & \multirow[b]{2}{*}{ ANOVA P-value } \\
\hline & & $E E C$ & G3 & $E S C$ & $U E C$ & \\
\hline \multicolumn{7}{|l|}{$D N A$ repair proteins } \\
\hline \multirow[t]{2}{*}{ MLH1 } & Positive & $43 / 5283 \%$ & $8 / 1457 \%$ & $26 / 2796 \%$ & $14 / 2070 \%$ & $P<0.05$ \\
\hline & Negative & $9 / 5217 \%$ & $6 / 1443 \%$ & $1 / 274 \%$ & $6 / 2030 \%$ & \\
\hline \multirow[t]{2}{*}{ PMS2 } & Positive & $40 / 5277 \%$ & $9 / 1560 \%$ & $24 / 2692 \%$ & $12 / 1963 \%$ & NS \\
\hline & Negative & $12 / 5223 \%$ & $6 / 1540 \%$ & $2 / 268 \%$ & $7 / 1937 \%$ & \\
\hline \multirow[t]{2}{*}{ MSH2 } & Positive & $51 / 5494 \%$ & $12 / 1485 \%$ & $23 / 2688 \%$ & $18 / 1995 \%$ & NS \\
\hline & Negative & $3 / 546 \%$ & $2 / 1414 \%$ & $3 / 2611 \%$ & 1/19 5\% & \\
\hline \multirow[t]{2}{*}{ MSH6 } & Positive & $38 / 4290 \%$ & $10 / 1377 \%$ & $21 / 2584 \%$ & $17 / 1894 \%$ & NS \\
\hline & Negative & 4/42 9\% & $3 / 1323 \%$ & $4 / 2516 \%$ & 1/18 6\% & \\
\hline \multirow[t]{2}{*}{ MSI } & Positive & $16 / 5529 \%$ & $9 / 1464 \%$ & $6 / 2722 \%$ & $8 / 2138 \%$ & $P<0.05$ \\
\hline & Negative & $39 / 5571 \%$ & $5 / 1436 \%$ & $21 / 2778 \%$ & $13 / 2162 \%$ & \\
\hline \multirow[t]{4}{*}{$\beta$-catenin } & Conserved & $22 / 5242 \%$ & $3 / 1520 \%$ & $9 / 2733 \%$ & $6 / 1932 \%$ & $P<0.05$ \\
\hline & Reduced & $17 / 5233 \%$ & $9 / 1560 \%$ & $18 / 2767 \%$ & $11 / 1958 \%$ & \\
\hline & Nuclear & $13 / 5225 \%$ & $3 / 1520 \%$ & $0 / 270$ & 1/19 5\% & \\
\hline & No expression & $0 / 520$ & $0 / 150$ & $0 / 270$ & $1 / 195 \%$ & \\
\hline \multirow[t]{2}{*}{ p53 } & Positive & $1 / 572 \%$ & $4 / 1527 \%$ & $14 / 2752 \%$ & $7 / 2133 \%$ & $P<0.001$ \\
\hline & Negative & $56 / 5798 \%$ & $11 / 1573 \%$ & $13 / 2748 \%$ & $14 / 2167 \%$ & \\
\hline \multirow[t]{3}{*}{ E-cadherin } & Conserved & $15 / 5328 \%$ & $2 / 1513 \%$ & $3 / 27$ 11\% & $0 / 210$ & $P<0.001$ \\
\hline & Reduced & $34 / 5364 \%$ & $10 / 1567 \%$ & $13 / 2748 \%$ & $7 / 2133 \%$ & \\
\hline & No expression & 4/53 7\% & $3 / 1520 \%$ & $11 / 2741 \%$ & $14 / 2167 \%$ & \\
\hline \multirow[t]{2}{*}{ P-cadherin } & Positive & $16 / 5330 \%$ & $11 / 1573 \%$ & $6 / 2722 \%$ & $1 / 205 \%$ & $P<0.001$ \\
\hline & Negative & $37 / 5370 \%$ & $4 / 1527 \%$ & $21 / 2778 \%$ & $19 / 2095 \%$ & \\
\hline \multirow[t]{3}{*}{ p120 } & Conserved & $31 / 5358 \%$ & $6 / 1540 \%$ & $8 / 2730 \%$ & $2 / 219 \%$ & $P<0.001$ \\
\hline & Reduced & $22 / 5341 \%$ & $9 / 1560 \%$ & $19 / 2770 \%$ & $11 / 2152 \%$ & \\
\hline & Cytoplasmic & $0 / 530$ & $0 / 150$ & $0 / 270$ & $8 / 2138 \%$ & \\
\hline \multirow[t]{2}{*}{ N-cadherin } & Positive & $13 / 5723 \%$ & $2 / 1513 \%$ & $3 / 2711 \%$ & $10 / 2050 \%$ & $P<0.05$ \\
\hline & Negative & $44 / 5777 \%$ & $13 / 1587 \%$ & $24 / 2789 \%$ & $10 / 2050 \%$ & \\
\hline \multirow[t]{2}{*}{ SPARC/osteonectin } & Positive & $5 / 4910 \%$ & $2 / 1414 \%$ & $2 / 277 \%$ & 8/18 44\% & $P<0.01$ \\
\hline & No expression & $44 / 4990 \%$ & $12 / 1486 \%$ & $25 / 2793 \%$ & $10 / 1856 \%$ & \\
\hline \multirow[t]{2}{*}{ FASCIN } & Positive & $23 / 5244 \%$ & $8 / 1553 \%$ & $17 / 2763 \%$ & $16 / 2176 \%$ & NS \\
\hline & Negative & $29 / 5256 \%$ & $7 / 1547 \%$ & $10 / 2737 \%$ & $5 / 2124 \%$ & \\
\hline \multirow[t]{2}{*}{ CAVEOLIN-1 } & Positive & $0 / 570$ & $0 / 150$ & $3 / 2711 \%$ & $2 / 219 \%$ & $P<0.05$ \\
\hline & Negative & $57 / 57100 \%$ & $15 / 15100 \%$ & $24 / 2789 \%$ & $19 / 2190 \%$ & \\
\hline \multirow[t]{2}{*}{ HMGA2 } & Positive & $0 / 570$ & $0 / 150$ & $7 / 2726 \%$ & $5 / 2124 \%$ & $P<0.001$ \\
\hline & Negative & $57 / 57100 \%$ & $15 / 15100 \%$ & $20 / 2774 \%$ & $16 / 2176 \%$ & \\
\hline \multirow[t]{2}{*}{ ZEB1 } & Positive & $0 / 570$ & $0 / 150$ & $0 / 270$ & $13 / 2162 \%$ & $P<0.001$ \\
\hline & Negative & $57 / 57$ 100\% & 15/15 100\% & $27 / 27100 \%$ & $8 / 2138 \%$ & \\
\hline
\end{tabular}

Microsatellite instability (MSI) was considered positive by the absence of expression of some mismatch repair (MMR) protein.

absence of expression of some of the mismatch repair protein) and p53 overexpression were associated in undifferentiated carcinomas and observed that only 2 of 21 samples showed both MSI and p53 overexpression (Supplementary Table 6).

Owing to lack of availability of further tissue microarray sections, we analyzed PTEN expression by immunohistochemistry on whole tissue sections only from the undifferentiated group. We observed only 5 out of 17 undifferentiated endometrial carcinomas with complete loss of PTEN expression. Two out of the five cases also showed mismatch repair deficiency and one showed p53 overexpression. We did not observe $\beta$-catenin nuclear expression in any areas of these undifferentiated endometrial carcinomas (data not shown).

\section{Absence of E-cadherin Expression is Characteristic of Undifferentiated Endometrial Carcinoma and it is Not Related to CDH1 Promoter Methylation}

To understand the molecular mechanism involved in the acquisition of an undifferentiated phenotype and based on our observation that E-cadherin expression is lost in some undifferentiated endometrial carcinomas, we analyzed E-cadherin expression in our series of endometrial carcinomas by immunohistochemistry and qRT-PCR. E-cadherin protein was not detected by immunohistochemistry in $\sim 70 \%$ of undifferentiated endometrial carcinomas and there was a sharp loss of this adhesion molecule in the remaining cases. This decrease was more significant when compared with other 
subtypes of endometrial carcinomas (Figure 1; Table 1), especially endometrioid endometrial carcinomas. Similar results were obtained by qRT-PCR, whereby undifferentiated endometrial carcinomas showed the weakest CDH1 mRNA expression (Figure 2a), presenting a 10.4-fold decrease compared with endometrial serous carcinomas, a 7.3-fold reduction compared with grade 3 endometrioid samples, and a 7.2-fold decrease with respect to grade $1 / 2$ endometrioid endometrial carcinomas $(P<0.001)$.

As promoter hypermethylation is known to silence CDH1 expression in other tumor types like breast cancer, ${ }^{15}$ we explored whether the $\mathrm{CDH} 1$ promoter was more strongly hypermethylated in undifferentiated endometrial carcinomas than in the other tumor types examined. By using a highly sensitive MassARRAY quantitative DNA methylation assay, we did not observe differences in DNA methylation levels of $\mathrm{CDH} 1$ promoter between undifferentiated and grade $1 / 2$ endometrioid endometrial carcinomas, the latter representing the tumor type that most strongly expressed E-cadherin. In fact, the absolute levels of $\mathrm{CDH} 1$ promoter methylation were very low (average of $18.5 \%$ in undifferentiated and $14.6 \%$ in endometrioid endometrial carcinomas), indicating a very modest (if any) regulation of $\mathrm{CDH} 1$ expression in undifferentiated endometrial carcinoma through this mechanism (Figure $2 \mathrm{~b}$ and $\mathrm{c}$ ).

\section{ZEB1 is Frequently Overexpressed in Undifferentiated Endometrial Carcinomas but not in other Histological Subtypes of Endometrial Carcinomas}

As expression of E-cadherin in undifferentiated endometrial carcinomas is reduced in the absence of $C D H 1$ promoter hypermethylation, we assessed the expression of several transcription factors that suppress E-cadherin expression by qRT-PCR, such as Snail1, Slug/Snail2, ZEB1, and ZEB2. Furthermore, we analyzed the expression of HMGA2, a nuclear factor that modulates E-cadherin expression through Snail1 and Slug/Snail2. Although we did not observe significant differences in the expression of these genes among the different histotypes, increased expression of ZEB1 in undifferentiated
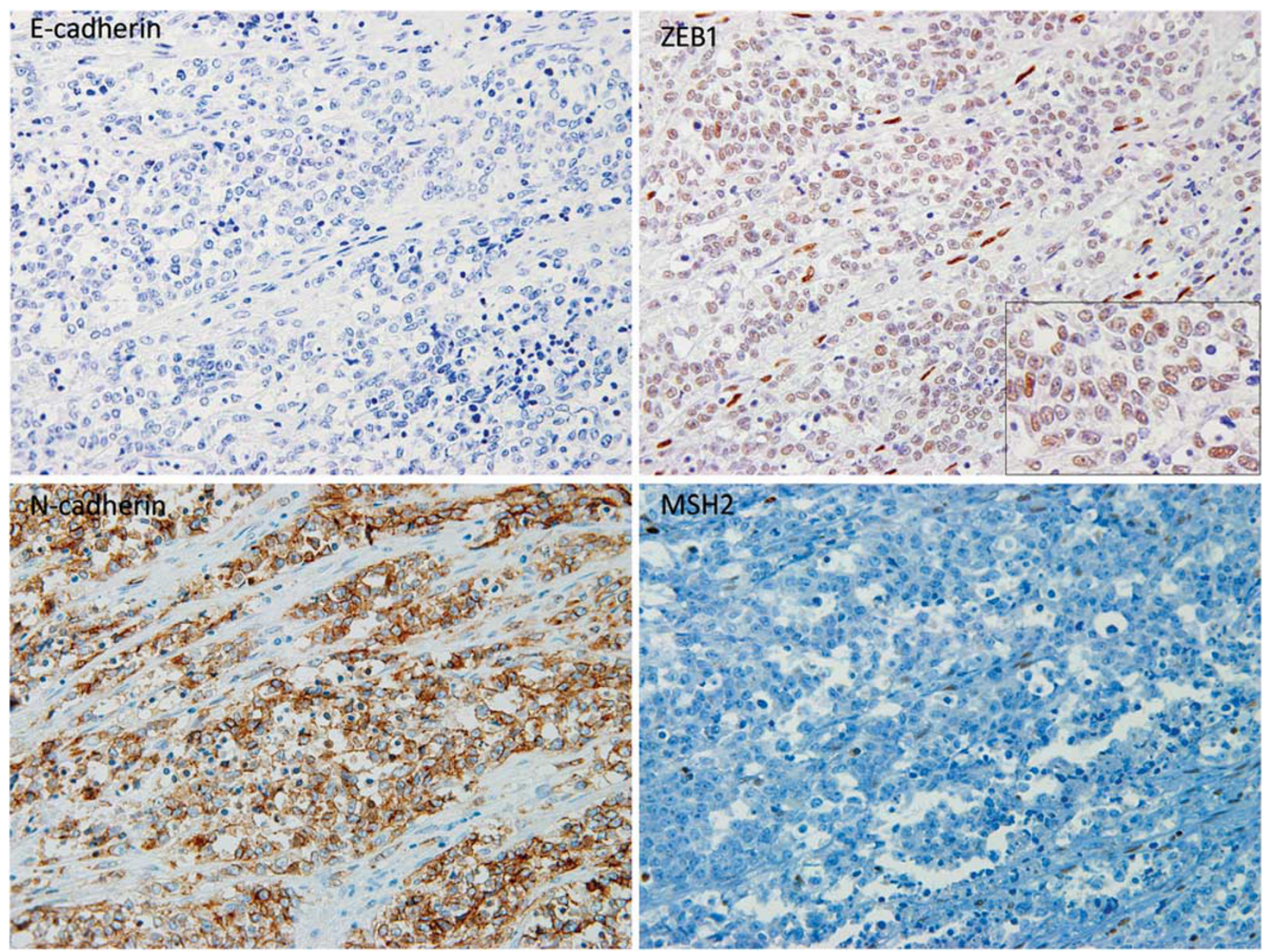

Figure 1 Immunohistochemistry on a formalin-fixed paraffin-embedded sample of undifferentiated endometrial carcinoma of E-cadherin $(\times 40)$; ZEB1 (this sample was described as low intensity in $30 \%$ of cells) $(\times 40)$, the inset shows a group of cells with nuclear expression of ZEB1; N-cadherin $(\times 40)$ and MSH2 $(\times 40)$. 

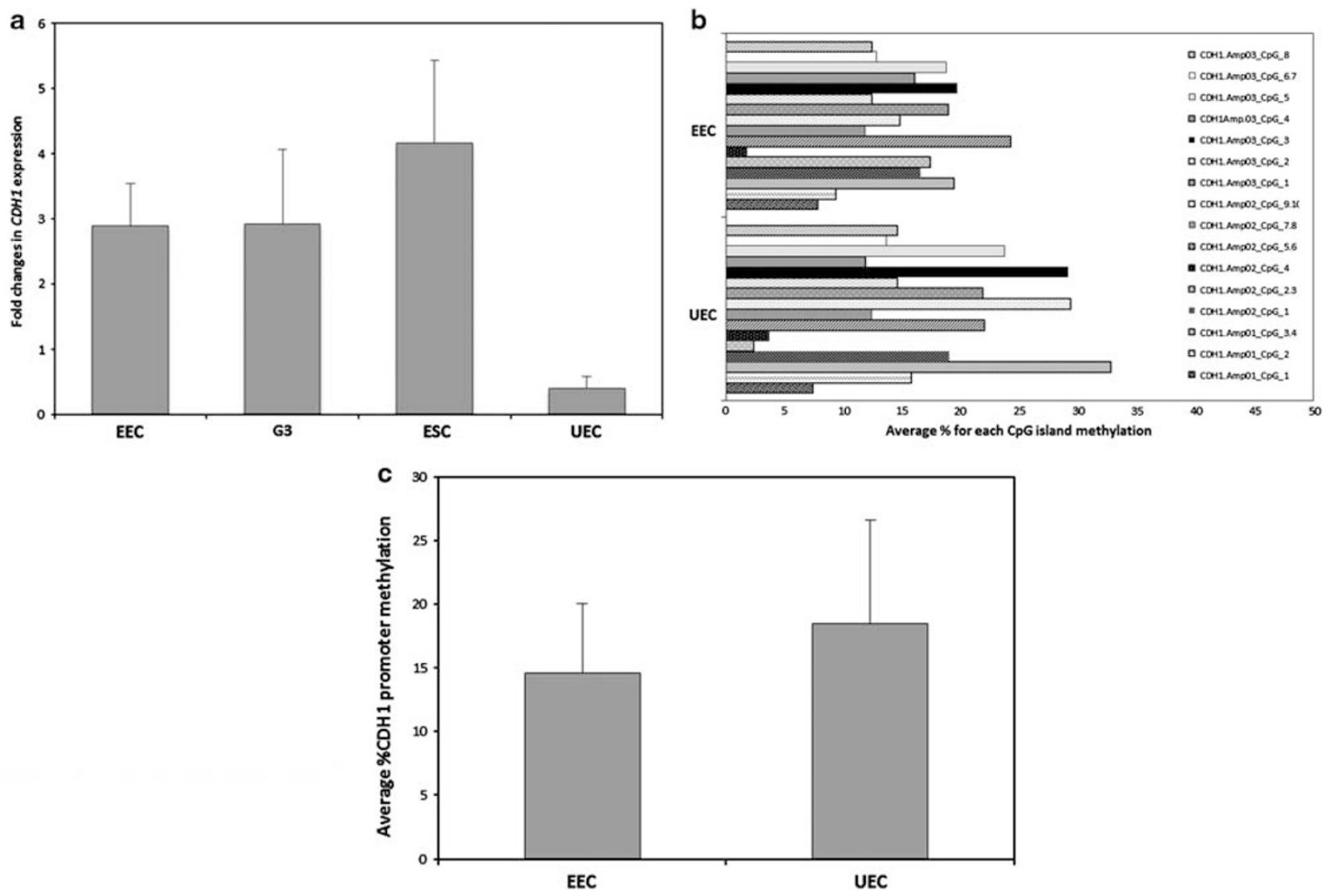

Figure 2 Decrease of $E$-cadherin expression in undifferentiated endometrial carcinoma (UEC). (a) Differences in $C D H 1$ expression among different groups of endometrial carcinoma (low-grade endometrioid endometrial carcinoma (EEC), high-grade EEC, endometrial serous carcinoma (ESC), and UEC) by qRT-PCR $(P<0.001)$. (b) Quantitative DNA methylation analysis of 22 CpG islands at $C D H 1$ promoter region, comparison between UEC and EEC. (c) Average percentage of DNA methylation levels of CDH1 promoter for the two groups.

endometrial carcinomas was nearly significant (data not shown). In addition, when determining correlations between E-cadherin expression and expression of E-cadherin repressors, the only correlation that was close to being significant was that between $E$-cadherin and $Z E B 1$ expression $(P=0.055)$.

Taking into account these results and the fact that qRT-PCR does not discriminate between gene expression in different tissue compartments (neoplastic epithelial cells versus stroma), we examined the distribution of these proteins by immunohistochemistry using commercial antibodies that work well in formalin-fixed paraffin-embedded tissue. We observed nuclear ZEB1 expression in $>60 \%$ of undifferentiated endometrial carcinomas but not in any of the other histotypes, although ZEB1 detected in stromal cells in all tumor samples served as a positive internal control (Figure 1, Table 1). The average proportion of cells expressing ZEB1 among undifferentiated carcinoma samples was about $26 \%$ (ranging from 5-60\%), which represents more than focal expression in most cases. HMGA2 was present in approximately $25 \%$ of undifferentiated and endometrial serous carcinomas but not in grade $1 / 2$ or grade 3 endometrioid endometrial carcinomas
(Table 1). Moreover, all HMGA2-positive undifferentiated endometrial carcinomas also expressed ZEB1.

\section{Downregulation of the miR-200 Family of miRNAs in Undifferentiated Endometrial Carcinomas}

We assessed all the endometrial carcinoma histotypes for the expression of miR-200s, a family of miRNAs that is composed of two clusters: miR200a/miR-200b/miR-429 on chromosome 1p36 and miR-200c/miR-141 on chromosome 12p13. All miR-200 family members were differentially expressed in undifferentiated carcinomas (Figure 3) when compared with: grade $1 / 2$ endometrioid endometrial carcinomas, 11.4-93.8-fold changes depending on the specific miRNA $(P<0.01)$, grade 3 endometrioid endometrial carcinomas, 3.23-6.1fold changes depending on the specific miRNA $(P<0.05)$, and endometrial serous carcinomas, 2.33.3-fold changes depending on the specific miRNA, except for miR-429 $(P<0.05)$. Correlation analysis showed that E-cadherin expression was directly correlated with that of all members of the miR-200 


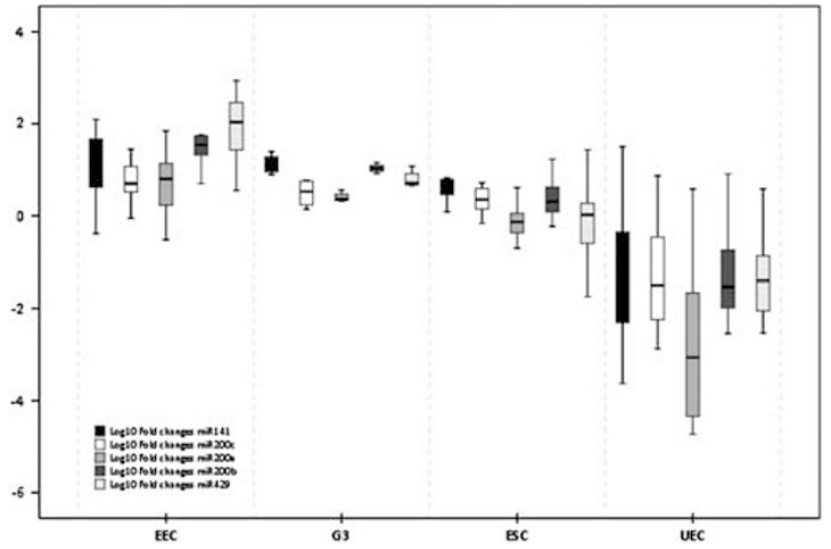

Figure 3 Analysis of miR-200s expression in endometrial carcinomas. Quantitative expression analysis of miR-200 family among different subtypes of endometrial carcinoma (low-grade endometrioid endometrial carcinoma (EEC), high-grade EEC, endometrial serous carcinoma (ESC), and undifferentiated endometrial carcinoma (UEC)).

family $(P<0.001)$, while significant inverse correlations were also found when the expression of each miRNA and ZEB1 expression was compared $(P<0.05$; Figure 4).

\section{Undifferentiated Endometrial Carcinomas Express an Epithelial-to-Mesenchymal Transition Phenotype}

Loss of E-cadherin, downregulation of miR-200s, and ZEB1 expression in endometrial carcinosarcomas ${ }^{16}$ is associated with an epithelial-to-mesenchymal transition phenotype that is characterized by changes in the expression of catenins, cadherin switching, and the expression of mesenchymal markers. Although undifferentiated endometrial carcinomas lacked any morphological evidence of mesenchymal differentiation, we detected an immunohistochemical profile that was similar to that seen in tumors with an epithelialto-mesenchymal transition phenotype. Thus, in addition to reduced expression of the epithelial E- and P-cadherins, undifferentiated endometrial carcinomas often expressed the mesenchymal N-cadherin (Figure 1). Moreover, abnormal cytoplasmic accumulation of p120, which is characteristic of mesenchymal cells, was observed in nearly $40 \%$ of undifferentiated carcinomas but not in the other histotypes. The mesenchymal markers osteonectin and fascin were also more frequently expressed in undifferentiated carcinomas than in the other types of endometrial tumors (44 and $76 \%$, respectively) (Table 1).

\section{Discussion}

Previous studies have demonstrated that undifferentiated endometrial carcinomas have reproducible morphological and immunohistochemical features, and an almost uniformly poor clinical outcome..$^{1,2,4}$ In this study, we investigated the molecular changes associated with undifferentiated endometrial carcinoma development, as well as those involved in tumor dedifferentiation.

Approximately $50 \%$ of undifferentiated endometrial carcinomas are associated with a differentiated component, most commonly grade $1 / 2$ endometrioid endometrial carcinoma, and such cases have been termed 'dedifferentiated carcinomas' ${ }^{3}$ or 'combined undifferentiated and differentiated carcinomas." ${ }^{1}$ These morphological features suggest that some undifferentiated carcinomas derive from endometrioid endometrial carcinomas and that molecular changes characteristic of endometrioid tumors are likely to be found in undifferentiated endometrial carcinomas. In this regard, we confirmed previous observations ${ }^{1,17}$ by demonstrating that the presence of DNA mismatch repair abnormalities was characteristic of undifferentiated endometrial carcinomas. This molecular alteration has been observed in $20-30 \%$ of EECs in a different series ${ }^{18}$ and it was present in nearly $40 \%$ of undifferentiated endometrial carcinomas in the present series. Although this series comprised 16 tumors previously analyzed, the frequency of mismatch repair abnormalities in new cases $(40 \%$ or $2 / 5)$ was similar to that of the prior study (37\% or 6/16). Mismatch abnormalities in undifferentiated endometrial carcinomas were first reported in sporadic tumors ${ }^{17}$ and it was subsequently observed that undifferentiated endometrial carcinomas can also occur in Lynch syndrome. ${ }^{1}$ In the present series, one patient showed loss of MSH2 and MSH6 expression in her undifferentiated endometrial carcinoma and is considered to be a presumptive Lynch syndrome patient. It is interesting to note that as reported previously, ${ }^{19}$ grade 3 endometrioid endometrial carcinomas studied here also showed a high frequency of mismatch repair abnormalities.

Nuclear expression of $\beta$-catenin is observed in $20-30 \%$ of endometrioid carcinomas yet it was only seen in one undifferentiated endometrial carcinoma, suggesting little relevance of the WNT pathway in the development of these tumors. This is consistent with the observation that endometrioid endometrial carcinomas with CTNNB1 abnormalities represent a subset of endometrial tumors with specific morphological features (frequent morular metaplasia) and with a relatively good prognosis. We found p53 to be expressed in 2,27 , and $52 \%$ of grade $1 / 2$ endometrioid endometrial carcinomas, grade 3 endometrioid endometrial carcinomas, and endometrial serous carcinomas, respectively, similar to previous reports. ${ }^{20,21}$ However, this was the first study to assess p53 expression in undifferentiated endometrial carcinomas, showing a frequency of overexpression $(33 \%)$ similar to that observed in grade 3 endometrioid carcinomas $(27 \%)$. Interestingly, only two of our seven p53-positive undifferentiated endometrial carcinomas also 


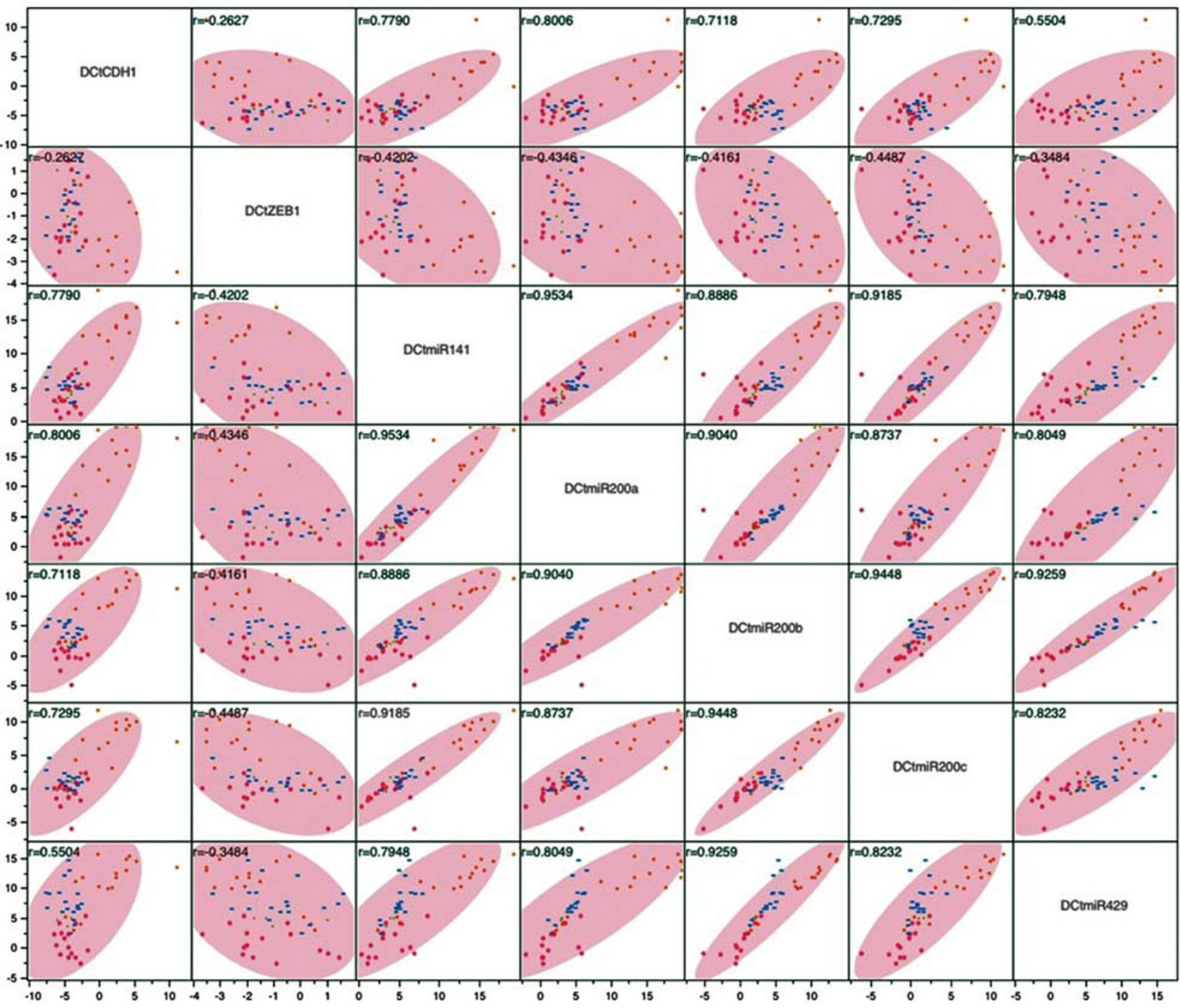

Figure 4 Correlations between $C D H 1$ and ZEB1 with each member of miR-200 family of miRNAs among samples. All correlations between $C D H 1$ and miR-200 expression were statistically significant at $P<0.001$. In the case of $Z E B 1$ and miR-200s, we obtained significant correlations at $P<0.05$. Red points represent samples of low-grade endometrioid endometrial carcinoma (EEC), green points represent high-grade EEC (G3), blue points are used for endometrial serous carinoma (ESC), and orange points show sample distribution of undifferentiated endometrial carcinoma (UEC).

showed mismatch repair alterations and none displayed nuclear $\beta$-catenin expression, as it has also been reported in grade 3 endometrioid endometrial carcinomas. ${ }^{20}$ While we cannot exclude other molecular changes characteristic of an endometrioid phenotype, this observation suggests that different molecular pathways are involved in undifferentiated endometrial carcinoma development. It is interesting to note that only 5 out of 21 undifferentiated carcinomas in this series showed complete absence of PTEN expression and that in three cases this alteration coexisted with mismatch deficiency and/or p53 overexpression. These findings may reflect the pathogenesis of undifferentiated endometrial carcinomas, some arising from lowgrade endometrioid endometrial carcinomas, while others can be considered 'de novo' cases derived from alterations in TP53.
One of the most remarkable findings of this study was the high incidence of complete loss of E-cadherin expression in undifferentiated endometrial carcinomas. E-cadherin was not detected in almost $70 \%$ of undifferentiated carcinomas and its expression was dramatically reduced in the remaining $30 \%$ of these tumors, while loss of E-cadherin expression was very uncommon in grade $1 / 2$ endometrioid endometrial carcinomas. There are multiple mechanisms that downregulate E-cadherin in cancer, such as gene mutation, gene silencing by promoter methylation, or transcriptional repression. Although we did not explore mutations in the $C D H 1$ gene, they are infrequent in human carcinomas and mostly restricted to a few histological types like lobular breast carcinoma and diffuse type gastric cancer. ${ }^{22}$ Using a qualitative approach, we and others $^{23,24}$ reported that $C D H 1$ promoter is 
hypermethylated in about $20 \%$ of endometrial carcinomas and that this is not associated with the histological type. In the quantitative analysis of CDH1 promoter methylation performed in this study, we found only weak methylation among the different histological types analyzed with no significant differences. These results indicated that CDH1 promoter hypermethylation was not a major determinant of the diminished E-cadherin expression in undifferentiated endometrial carcinoma.

Our results suggest that E-cadherin loss in undifferentiated carcinomas was partly due to $Z E B 1$ overexpression. Indeed, we found ZEB1 mRNA expression to be inversely correlated with E-cadherin levels. ZEB1 is a zinc-finger transcriptional factor that represses $E$-cadherin expression by acting on specific E-boxes in its promoter. ${ }^{25}$ Our immunohistochemical results confirmed previous observations that ZEB1 expression is confined to the stroma in normal endometrium and most endometrial carcinomas. In addition, we found $Z E B 1$ to be expressed in $5-60 \%$ of neoplastic cells in undifferentiated endometrial carcinomas but not in the other carcinoma histotypes. There are only two previous studies investigating ZEB1 expression in endometrial carcinoma. In one report, ZEB1 expression was found in 4 grade 3 tumors from a group of 15 grade $1 / 2$ and grade 3 endometrioid endometrial carcinomas studied. There was also epithelial ZEB1 expression in 4 and 5 out of 12 endometrial serous carcinomas and 29 carcinosarcomas, respectively. ${ }^{26}$ A larger study (88 endometrial carcinomas) from the same group included a expanded number of endometrioid endometrial carcinomas (71 grade $1 / 2$ and 17 grade 3 ), but also 3 undifferentiated endometrial carcinomas and 12 endometrial carcinomas with a serous component. ZEB1 expression was only observed in three grade 3 endometrioid endometrial carcinomas, ${ }^{27}$ although it was not specified whether the ZEB1-positive grade 3 endometrioid tumors were the same in the two studies. Differences between their series and the series presented herein might be due to different criteria to diagnose undifferentiated endometrial carcinomas.

We do not know the mechanisms underlying ZEB1 overexpression in undifferentiated endometrial carcinomas. The TGF $\beta$ pathway modulates ZEB1 expression, and although we did not observe any differences in TGF $\beta 1$ and TGF $\beta 2$ mRNA expression among the different histotypes, we cannot exclude differences in protein expression/ activation. Indeed, for the first time we noted that 5 out of $21(24 \%)$ undifferentiated carcinomas expressed HMGA2, a well-established target of the TGF $\beta$ pathway. Interestingly, all five HMGA2-positive tumors also expressed ZEB1, suggesting activation of the TGF $\beta$ pathway in some undifferentiated carcinomas. HMGA2 overexpression has been demonstrated in endometrial serous carcinomas and carcinosarcomas, ${ }^{28,29}$ but is found only infrequently in endometrioid endometrial carcinomas, as indicated here, even including poorly differentiated endometrioid tumors.

Wild-type p53 has recently been seen to fulfill a critical function in repressing the expression of $Z E B 1$ and ZEB2 through members of the miR-200 and miR192 families. ${ }^{30,31}$ Members of the miR-200 family are directly regulated by p53 at the transcriptional level and the loss of p53 is correlated with decreased miR-200c. Here, we demonstrated for the first time that a marked reduction of miR-200s was characteristic of undifferentiated endometrial carcinomas. Although low levels of miR-200s can produce $Z E B 1$ upregulation, the specific role of p53 in miR-200s/ZEB1 modulation in undifferentiated endometrial carcinomas is debatable as the number of these tumors with p53 overexpression was relatively low (33.3\%). Furthermore, we did not observe ZEB1 upregulation in endometrial serous carcinomas, which exhibited a high prevalence of TP53 changes, but also higher levels of miR-200s than undifferentiated endometrial carcinomas.

We noted that miR-200 expression was associated with histotype and degree of differentiation. Indeed, the expression of miR-200s was high in grade $1 / 2$ endometrioid endometrial carcinomas, it decreased in grade 3 endometrioid carcinomas and endometrial serous carcinomas, and it was further reduced in undifferentiated endometrial carcinomas. These results are consistent with the proposed role of miR-200s in maintaining a differentiated epithelial phenotype. ${ }^{10,11,32}$ In a recent review, ${ }^{33}$ the regulation of the miR-200 family by estrogen receptor (ER)- $\alpha$ and estradiol was described and indeed, the proportion of cases and intensity of ER expression was lower in grade 3 endometrioid carcinomas and serous carcinomas than in low-grade endometrioid endometrial carcinomas. Moreover, only a small proportion of undifferentiated endometrial carcinomas $(\sim 10 \%)$ exhibited focal ER expression. ${ }^{1}$

A major consequence of miR-200s downregulation in endometrial carcinogenesis is the activation of epithelial-to-mesenchymal transition. ${ }^{16}$ Although we did not observe morphological evidence of a mesenchymal phenotype in undifferentiated endometrial carcinomas, as seen in carcinosarcomas, we found increased expression of some mesenchymal markers, such as N-cadherin, cytoplasmic p120, and osteonectin, in these tumors relative to other histotypes. These changes, together with E-cadherin downregulation, might confer increased cellular motility, invasiveness, and metastatic ability to undifferentiated carcinomas, that can relate to poor prognosis. The expression of both epithelial and mesenchymal markers in undifferentiated endometrial tumors indicates a high degree of cellular plasticity, as observed in cancer stem cells. Thus, our observation, together with the established role of ZEB1/miR-200s in 'stemness', suggests that undifferentiated endometrial carcinoma might be enriched in cancer stem cells. In fact, different 
studies have demonstrated that the balanced expression of ZEB factors and miR-200s not only controls epithelial-to-mesenchymal transition but also 'stemness'. ${ }^{34}$ Significantly, relevant molecular targets of the miR-200/ZEB1 loop include the stem cell-associated factors BMI1, SOX2, KLF4, ${ }^{14}$ and Notch signaling pathway. ${ }^{35}$

Summarizing, we demonstrated for the first time that absence or severe dowregulation of E-cadherin associated with ZEB1 overexpression is characteristic of undifferentiated endometrial carcinomas. As these alterations are very infrequently seen in grade 3 endometrioid endometrial carcinomas and can be evaluated by immunohistochemistry, our results have important implications for the differential diagnosis of both types of tumors, which show different prognosis.

\section{Acknowledgements}

We thank Ana Santos, Ma José Hernández, and Susana Ramiro for their excellent technical assistance. We would also like to acknowledge Dr David Huntman's and Osama Al-Agha's contributions during the early phases of this work. This work was supported by grants from the Instituto de Salud Carlos III (ISCIII; Grant No. PI080971) co-financed by the European Development Regional Fund, 'A way to achieve Europe' EDRF (Grant No. RD12/ 0036/0064); the Junta de Andalucía (Consejería de Salud, Grant No. PI0581/2009) to JP; grants FISPI10/ 00922, 2009SGR794, RD12/0036/0013, and by a grant from the Asociación Española Contra el Cáncer, Red de Grupos estables en Oncología (AECC-2011) to XM-G. LR-P is a PhD student recipient of a PFIS fellowship (Grant No. F109/ 00193). JD-M is a $\mathrm{PhD}$ researcher funded by the Consejería de Salud, Junta de Andalucía (PI0581/ 2009). MB is a researcher funded by the ISCIII-Red de Biobancos RD09/0076/00085 and MAC is a PhD researcher funded by the ISCIII (Grant No. RD06/ 0020/0013).

\section{Disclosure/conflict of interest}

The authors declare no conflict of interest.

\section{References}

1 Tafe LJ, Garg K, Chew I, et al. Endometrial and ovarian carcinomas with undifferentiated components: clinically aggressive and frequently underrecognized neoplasms. Mod Pathol 2010;23:781-789.

2 Altrabulsi B, Malpica A, Deavers MT, et al. Undifferentiated carcinoma of the endometrium. Am J Surg Pathol 2005;29:1316-1321.

3 Silva EG, Deavers MT, Bodurka DC, et al. Association of low-grade endometrioid carcinoma of the uterus and ovary with undifferentiated carcinoma: a new type of dedifferentiated carcinoma? Int J Gynecol Pathol 2006;25:52-58.

4 Silva EG, Deavers MT, Malpica A. Undifferentiated carcinoma of the endometrium: a review. Pathology 2007;39:134-138.

5 Giordano G, D’Adda T, Bottarelli L, et al. Two cases of low-grade endometriod carcinoma associated with undifferentiated carcinoma of the uterus (dedifferentiated carcinoma): a molecular study. Pathol Oncol Res 2012;18:523-528.

6 Yoshida S, Furukawa N, Haruta S, et al. Expression profiles of genes involved in poor prognosis of epithelial ovarian carcinoma: a review. Int J Gynecol Cancer 2009;19:992-997.

7 Moreno-Bueno G, Portillo F, Cano A. Transcriptional regulation of cell polarity in EMT and cancer. Oncogene 2008;27:6958-6969.

8 Peinado H, Olmeda D, Snail Cano A. Zeb and bHLH factors in tumour progression: an alliance against the epithelial phenotype? Nat Rev Cancer 2007;7:415-428.

9 Sarrio D, Rodriguez-Pinilla SM, Hardisson D, et al. Epithelial-mesenchymal transition in breast cancer relates to the basal-like phenotype. Cancer Res 2008;68:989-997.

10 Gregory PA, Bert AG, Paterson EL, et al. The miR-200 family and miR-205 regulate epithelial to mesenchymal transition by targeting ZEB1 and SIP1. Nat Cell Biol 2008;10:593-601.

11 Park SM, Gaur AB, Lengyel E, et al. The miR-200 family determines the epithelial phenotype of cancer cells by targeting the E-cadherin repressors ZEB1 and ZEB2. Genes Dev 2008;22:894-907.

12 Burk U, Schubert J, Wellner U, et al. A reciprocal repression between ZEB1 and members of the miR-200 family promotes EMT and invasion in cancer cells. EMBO Rep 2008;9:582-589.

13 Bracken CP, Gregory PA, Kolesnikoff N, et al. A doublenegative feedback loop between ZEB1-SIP1 and the microRNA-200 family regulates epithelial-mesenchymal transition. Cancer Res 2008;68:7846-7854.

14 Brabletz S, Brabletz T. The ZEB/miR-200 feedback loop-a motor of cellular plasticity in development and cancer? EMBO Rep 2010;11:670-677.

15 Sebova K, Zmetakova I, Bella V, et al. RASSF1A and $\mathrm{CDH} 1$ hypermethylation as potential epimarkers in breast cancer. Cancer Biomark 2011;10:13-26.

16 Castilla MA, Moreno-Bueno G, Romero-Perez L, et al. Micro-RNA signature of the epithelial-mesenchymal transition in endometrial carcinosarcoma. J Pathol 2011;223:72-80.

17 Broaddus RR, Lynch HT, Chen LM, et al. Pathologic features of endometrial carcinoma associated with HNPCC: a comparison with sporadic endometrial carcinoma. Cancer 2006;106:87-94.

18 Peterson LM, Kipp BR, Halling KC, et al. Molecular characterization of endometrial cancer: a correlative study assessing microsatellite instability, MLH1 hypermethylation, DNA mismatch repair protein expression, and PTEN, PIK3CA, KRAS, and BRAF mutation analysis. Int J Gynecol Pathol 2012;31:195-205.

19 Karamurzin Y, Rutgers JK. DNA mismatch repair deficiency in endometrial carcinoma. Int J Gynecol Pathol 2009;28:239-255.

20 Alvarez T, Miller E, Duska L, et al. Molecular profile of grade 3 endometrioid endometrial carcinoma: is it a type I or type II endometrial carcinoma? Am J Surg Pathol 2012;36:753-761. 
21 Alkushi A, Kobel M, Kalloger SE, et al. Highgrade endometrial carcinoma: serous and grade 3 endometrioid carcinomas have different immunophenotypes and outcomes. Int J Gynecol Pathol 2010; 29:343-350.

22 Berx G, Becker KF, Hofler H, et al. Mutations of the human E-cadherin (CDH1) gene. Hum Mutat 1998;12:226-237.

23 Moreno-Bueno G, Hardisson D, Sarrio D, et al. Abnormalities of E- and P-cadherin and catenin (beta-, gamma-catenin, and p120ctn) expression in endometrial cancer and endometrial atypical hyperplasia. J Pathol 2003;199:471-478.

24 Banno K, Yanokura M, Susumu N, et al. Relationship of the aberrant DNA hypermethylation of cancerrelated genes with carcinogenesis of endometrial cancer. Oncol Rep 2006;16:1189-1196.

25 Grooteclaes ML, Frisch SM. Evidence for a function of CtBP in epithelial gene regulation and anoikis. Oncogene 2000;19:3823-3828.

26 Spoelstra NS, Manning NG, Higashi Y, et al. The transcription factor ZEB1 is aberrantly expressed in aggressive uterine cancers. Cancer Res 2006;66: 3893-3902.

27 Singh M, Spoelstra NS, Jean A, et al. ZEB1 expression in type I vs type II endometrial cancers: a marker of aggressive disease. Mod Pathol 2008;21:912-923.

28 McCluggage WG, Connolly LE, McBride HA, et al. HMGA2 is commonly expressed in uterine serous carcinomas and is a useful adjunct to diagnosis. Histopathology 2012;60:547-553.

29 Romero-Perez L, Castilla MA, Lopez-Garcia MA, et al. Molecular events in endometrial carcinosarcomas and the role of high mobility group AT-hook 2 in endometrial carcinogenesis. Hum Pathol 2013;44:244-254.

30 Kim T, Veronese A, Pichiorri F, et al. p53 regulates epithelial-mesenchymal transition through microRNAs targeting ZEB1 and ZEB2. J Exp Med 2011;208:875-883.

31 Chang CJ, Chao CH, Xia W, et al. p53 regulates epithelial-mesenchymal transition and stem cell properties through modulating miRNAs. Nat Cell Biol 2011;13:317-323.

32 Korpal M, Kang Y. The emerging role of miR-200 family of microRNAs in epithelial-mesenchymal transition and cancer metastasis. RNA Biol 2008;5: 115-119.

33 Cochrane DR, Cittelly DM, Richer JK. Steroid receptors and microRNAs: relationships revealed. Steroids 2011;76:1-10.

34 Wellner U, Schubert J, Burk UC, et al. The EMTactivator ZEB1 promotes tumorigenicity by repressing stemness-inhibiting microRNAs. Nat Cell Biol 2009;11:1487-1495.

35 Brabletz S, Bajdak K, Meidhof S, et al. The ZEB1/miR200 feedback loop controls Notch signalling in cancer cells. EMBO J 2011;30:770-782.

Supplementary Information accompanies the paper on Modern Pathology website (http://www.nature.com/ modpathol) 